\title{
The Analysis of the Thickness-predictive Model Based on the SVM
}

\author{
Xiu-ming Zhao ${ }^{1, a}$, Yan Wang ${ }^{2, b}$ and Zhimin $\mathrm{Bi}^{3, \mathrm{c}}$ \\ ${ }^{1}$ School of Control Science and Engineering, University of Jinan, Jinan250022, China \\ ${ }^{2}$ School of Control Science and Engineering, University of Jinan, Jinan250022, China \\ ${ }^{3}$ School of Control Science and Engineering, University of Jinan, Jinan250022, China \\ aemail: jd_zxm@163.com, bemail:272508613@qq.com, 'email:969543630@qq.com
}

Keywards: hot rolling; thickness prediction; SVM; AGC;

\begin{abstract}
This paper presents a method which improves the thickness of the prediction model. At the same time, it builds up a SVM prediction model based on MATLAB simulation experiment. By comparing the simulation curves of the mechanism model and SVM regression prediction, it apparently can be seen that thickness-output precision effectively improved, which is good to on-line identification of the thickness control.
\end{abstract}

\section{Introduction}

Metallurgical industry at home and abroad pay more attention to the quality of the plate strip, especially the dimensions, the thickness accuracy has become one of the important indicators. And precision problem of thickness control has restricted the product quality improvement. Through improving the predictive accuracy of strip thickness and achieving more precise control, the plate band steel production play a crucial role.

The thickness predicted system is the important method of plate strip thickness accuracy based on mechanism model, its purpose is to realize the precise control online. However, environment is more complex due to the continuous strip production, the mechanism model is difficult to establish. The mechanism of the model we used is got after ignoring and simplifying some of the factors .In continuous production, rolling technology is more complex and include unknown factors of the uncertainty, these factors are difficult to establish accurate mathematical model. Therefore, the use of a large amount of wealth in the process of actual production data modeling and control become inevitable choice.

\section{The SVM prediction model based on the thickness}

Vapnik put forward a kind of novel machine learning method of support vector machine (SVM): the basic idea of SVM input sample space is mapped to high-dimensional feature space by using kernel functions, to find an optimal classification plane in high dimensional space. Through SVM processing, to obtain the nonlinear mapping relationship between variable input and variable output. It is often used when the support vector machine (SVM) algorithm in dealing with small sample, nonlinear and function fitting problems.In fact, the algorithm of support vector machine (SVM) is a convex quadratic optimization problem, the solution is global optimal solution. At present, support vector machine (SVM) get more successful applications in bioinformatics face and gesture recognition, text classification and so on.

Support vector machine (SVM) regression algorithm of linear and nonlinear. For linear regression problem, given the training set $\left\{\left(x_{1}, y_{1}\right),\left(x_{2}, y_{2}\right), \cdots,\left(x_{i}, y_{i}\right)\right\}$, Among them $x_{i} \in R^{n}$, $y \in R$, Regression linear function can be used by $f(x)=\omega^{T} x+b$. It can be got the best regression function by solving the minimum value, as formula(1). 


$$
\varphi\left(\omega, \xi, \xi^{*}\right)=\frac{1}{2}\|\omega\|^{2}+C\left(\sum_{i=1}^{t} \xi_{i}+\sum_{i=1}^{t} \xi^{*}\right)
$$

where $\mathrm{C}$ is Penalty factor, $\xi, \xi^{*}$ is Respectively upper and lower bounds of slack variable. The constraint as formula(2).

$$
\left\{\begin{array}{l}
f\left(x_{i}\right)-y_{i} \leq \xi_{i}^{*}+\varepsilon \\
y_{i}-f\left(x_{i}\right) \leq \xi_{i}+\varepsilon, \quad i=1,2, \cdots k \\
\xi, \xi^{*} \geq 0
\end{array}\right.
$$

Linear separable problem is also a convex quadratic optimization problem. To solve the problem, we introduce the Lagrange function, as formula(3).

$$
\begin{aligned}
& L\left(\omega, b, \xi, \xi^{*}, a, a^{*}, \gamma, \lambda^{*}\right)= \\
& \frac{1}{2}\|\omega\|^{2}-C \sum_{i=1}^{t}\left(\xi_{i}+\xi_{i}^{*}\right)-\sum_{i=1}^{t} a_{i}\left[\xi+\varepsilon-y_{i}+f\left(x_{i}\right)\right] \\
& -\sum_{i=1}^{t} a_{i}\left[\xi_{i}^{*}+\varepsilon+y_{i}-f\left(x_{i}\right)\right]-\sum_{i=1}^{t} \xi_{i} \gamma_{i}+\xi_{i}^{*} \lambda_{i}^{*}
\end{aligned}
$$

where $a_{i}, a_{i}^{*} \geq 0, \quad \gamma_{i}, \lambda_{i}^{*} \geq 0, \quad i=1, \cdots, k$. The Lagrange function $\mathrm{L}$ solve the minimum value answering to $\omega, b, \xi_{i}, \xi_{i}^{*}$, as formula(4).

$\frac{\partial L}{\partial \omega}=0, \frac{\partial L}{\partial b}=0, \frac{\partial L}{\partial \xi_{i}}=0, \frac{\partial L}{\partial \xi_{i}^{*}}=0$

It can be described by the formula above, as formula(5).

$$
\left\{\begin{array}{l}
\sum_{i=1}^{t}\left(a_{i}-a_{i}^{*}\right)=0 \\
\omega=\sum_{i=1}^{t}\left(a_{i}-a_{i}^{*}\right) x_{i} \\
C-a_{i}-\gamma_{i}=0
\end{array}\right.
$$

It can get a convex quadratic optimization problem dual form by pluging the above formula in the Lagrange function, as formula(6).

$$
\begin{aligned}
& \omega\left(a, a^{*}\right)=-\frac{1}{2} \sum_{i, j=1}^{t}\left(a_{i}-a_{i}^{*}\right)\left(a_{j}-a_{j}^{*}\right)\left(x_{i} \cdot x_{j}\right) \\
& +\sum_{i=1}^{t}\left(a_{i}-a_{i}^{*}\right) y_{i}-\sum_{j=1}^{t}\left(a_{i}-a_{i}^{*}\right) \varepsilon
\end{aligned}
$$

As the constraint conditions in formula(7).

$$
\sum_{i=1}^{t}\left(a_{i}-a_{i}^{*}\right)=0, \quad 0 \leq a_{i}, \quad a_{i}^{*} \leq C
$$

Regression function can be obtained by calculating the value of the formula 5 and pluging in the formula (8).

$$
f(x)=\sum_{i=1}^{n}\left(a_{i}-a_{i}^{*}\right)\left(x_{i}, x\right)+b
$$

For nonlinear regression problem, it need to complete the following two aspects: first, it will realize the mapping from the original sample space to a high-dimensional space using a nonlinear mapping function. Second, it should analyze by using the method of linear regression in the high dimension space. So the key of dealling with nonlinear regression problem is to ask the mapping function, thus the problem become optimize the formula(3) of under the constraint conditions as formula(9). 


$$
\begin{aligned}
& \omega\left(a, a^{*}\right)=-\frac{1}{2} \sum_{i, j=1}^{t}\left(a_{i}-a_{i}^{*}\right)\left(a_{j}-a_{j}^{*}\right) k\left(x_{i} \cdot x_{j}\right) \\
& +\sum_{i=1}^{t}\left(a_{i}-a_{i}^{*}\right) y_{i}-\sum_{j=1}^{t}\left(a_{i}-a_{i}^{*}\right) \varepsilon
\end{aligned}
$$

Where $\omega=\sum_{i=1}^{t}\left(a_{i}-a_{i}^{*}\right) \varphi\left(x_{i}\right)$, The regression linear function can be used by $f(x)=\sum_{i=1}^{t}\left(a_{i}-a_{i}^{*}\right) k\left(x_{i}, x\right)+b$

Kernel function of support vector machine use the gaussian kernel function and polynomial kernel function and sigmoid kernel function, etc.

Gaussian kernel function: $\quad K\left(x, x_{i}\right)=\exp \left[-\left\|x-x_{i}\right\|^{2} / 2 \sigma^{2}\right]$

Polynomial function: $\quad K\left(x, x_{i}\right)=\left(x . x_{i}+1\right)^{d}$

Sigmoid kernel function: $\quad K\left(x, x_{i}\right)=\tanh \left[\alpha\left(x . x_{i}\right)+C\right]$

$$
K\left(x, x_{i}\right)=\frac{1}{1+\exp \left[\alpha\left(x . x_{i}\right)+C\right]}
$$

\section{The SVM prediction model based on matlab simulation experiment}

It should establish strip finishing exit thickness prediction model by Libsvm toolkit. Algorithm process as shown in figure 1.

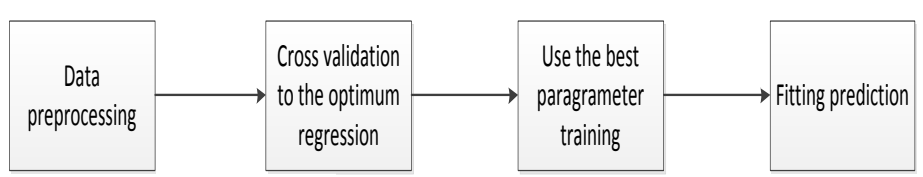

Figure 1 the SVM prediction model algorithm flow chart

The simulation of MATLAB code:

$\% \%$ to find the best parameter c and g of regression problems:

[bestmse,bestc,bestg] = SVMcgForRegress(TS,TSX,-8,8,-8,8);

[bestmse,bestc,bestg] = SVMcgForRegress(TS,TSX,-4,4,-4,4,3,0.5,0.5,0.05);

$\% \%$ it shoukd use the best parameters $\mathrm{c}$ and $\mathrm{g}$ for training of SVM:

cmd = ['-c ', num2str(bestc), ' -g ', num2str(bestg) , ' -s 3 -p 0.01'];

model $=$ svmtrain(TS, TSX,cmd);

$\% \%$ the SVM regression prediction:

[predict,mse] = svmpredict(TS1,TSX1, model);

predict = mapminmax('reverse',predict',TSps);

The simulation results and analysis: 

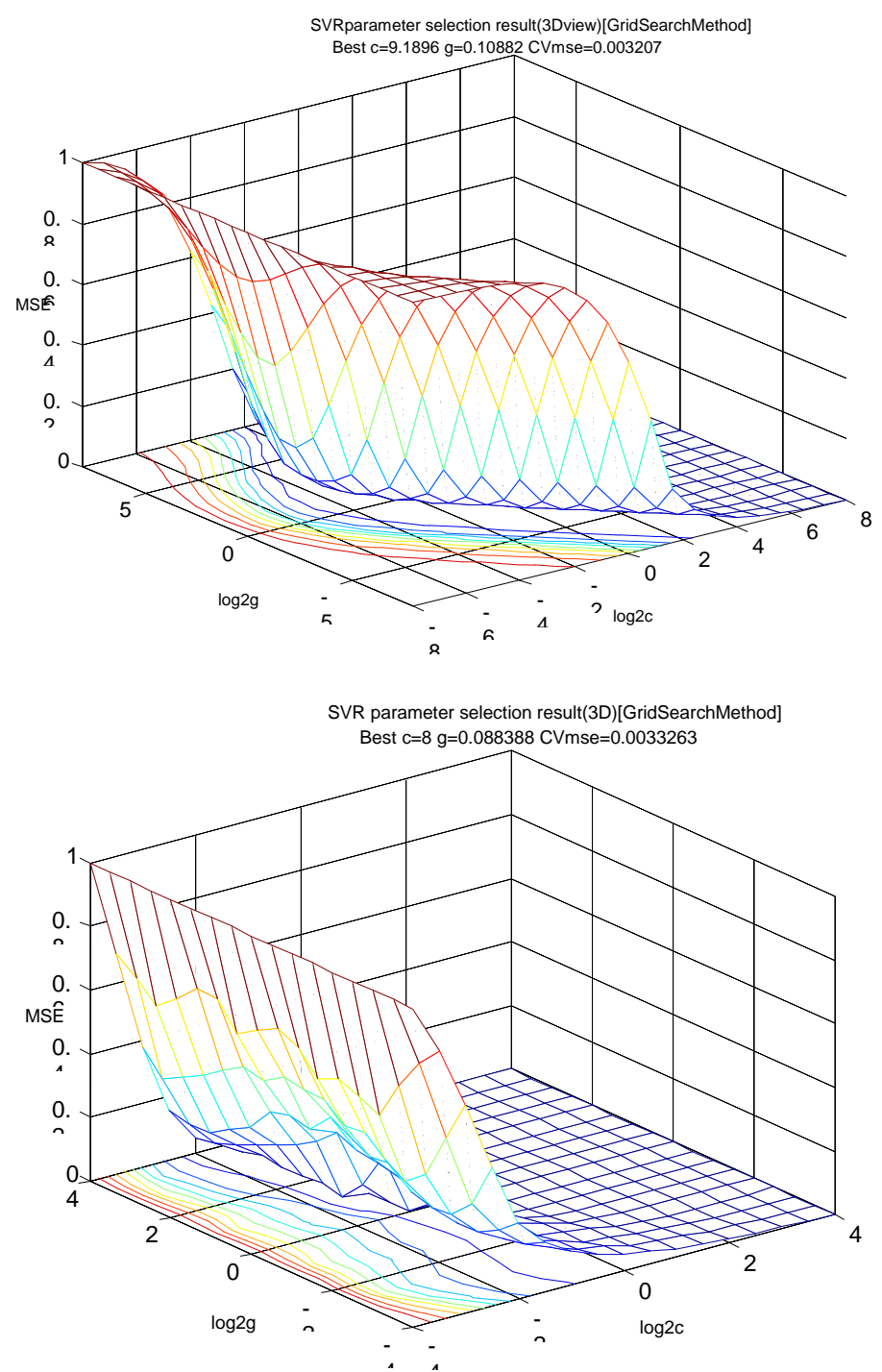

Figure 2 Parameter selection result figure of rough and fine

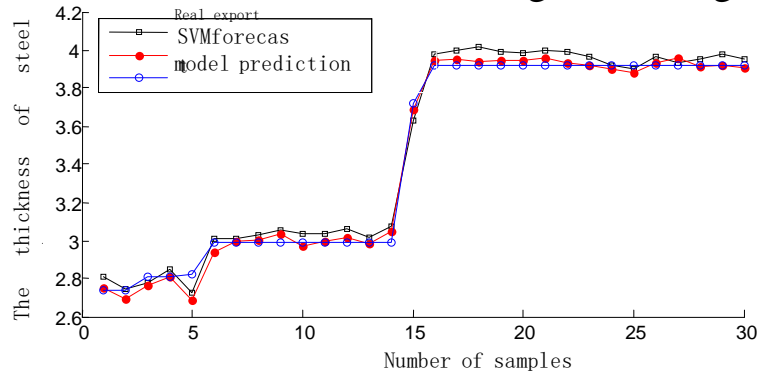

Figure 3 Thickness output curve of the prediction and the reality

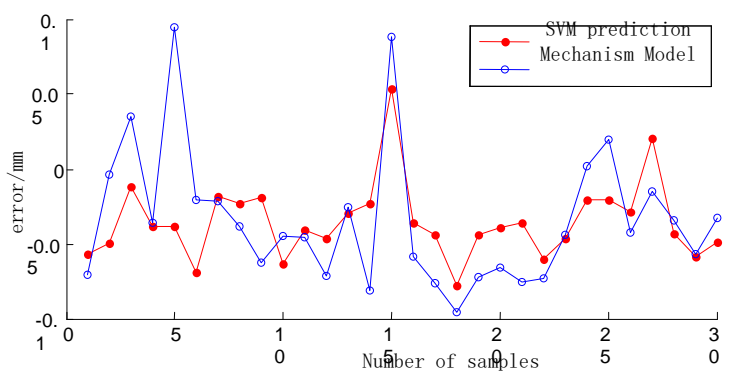

Figure 4 Thickness output error curve of the prediction and the reality 


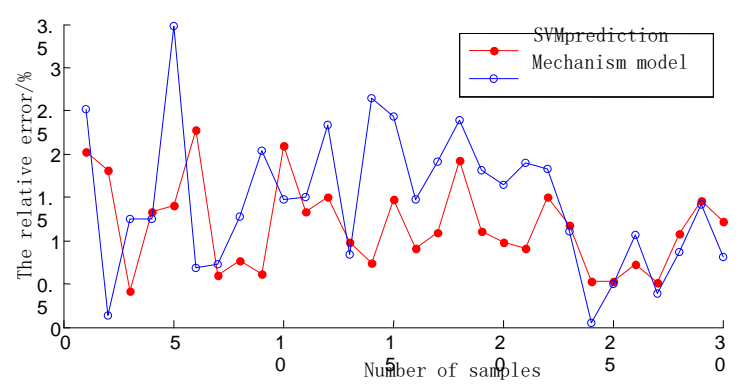

Figure 5 thickness output absolute error curve of the prediction and the reality

By the simulation results contrast curve can be concluded that the prediction algorithm based on support vector machine (SVM) can be a very good prediction, the export of steel strip thickness and comparison with actual measurement results of error and absolute error generally smaller than mechanism model. Description based on support vector machine (SVM) algorithm in optimal problems of strip steel thickness control has significant advantages.

\section{Conclusion}

This paper establishes the prediction model based on SVM and thickness. But for complex actual rolling process, it's just taken the first step and still have a lot of work to do.It can do layered with a large number of historical data from the field, and realize the offline collection system of layered modeling.

\section{Reference}

[1] dong-dong liu, Wang Yan. Based on RBF neural network strip finishing thickness prediction [J]. Journal of jinan university: natural science edition, 2006, 20 (4) : 312-315.

[2] ying-ying liu, Wang Yan. The thickness of the hot rolled recognition and intelligent forecast [J]. Journal of jinan university: natural science edition, 2014,28 (2) : 110-113.

[3] Ibtissem Ladlani, Larbi Houichi, Lakhdar Djemili, et al. Modeling daily reference evapotranspiration (ET0) in the north of Algeria using generalized regression neural networks (GRNN) and radial basis function neural networks (RBFNN): a comparative study[J]. Springer-Verlag,2012,118:163-178.

[4] Williams James Michael Zhang Deyong Li Shucai, etc. The genetic - RBF neural network based on rough set theory in the application of rock burst prediction [J]. Rock and soil mechanics, 2012, 31 (1) : 270-276.

[5] liou j w, facing south, Roger wang. Based on the data driven modeling and control of strip thickness

[6] Method [J]. Journal of Beijing university of technology, 2012, 20 (12) : 1913-1920.

[7] Baihua Zhang. Prediction of Freight Ability in Country Base on GRNN[J]. IFIP International Federation for Information Processing,2011,346:525-531. 